PACS: $73.40 ; 78.65 ; 77.30$

\title{
THEORY OF ENERGY TRANSFER OF ELECTRON EXCITATION: KINETICS OF EXCITON LUMINESCENCE IN A THREE-LAYER SYSTEMS
}

\author{
Sergey I. Pokutnyi \\ Illichivsk Educational Research Center, \\ I. I. Mechnikov Odessa National University \\ 17a, Danchenka str., Illichivsk, Odessa reg. \\ 68001 Ukraine; E-mail: univer@ivt.ilyichevsk.odessa.ua
}

\begin{abstract}
THEORY OF ENERGY TRANSFER OF ELECTRON EXCITATION: KINETICS OF EXCITON LUMINESCENCE IN A THREE-LAYER SYSTEMS
\end{abstract}

Sergiy. I. Pokutnyi

The decay kinetics of exciton luminescence in impurity molecules in a three-layer system near the interface of dielectric media in the presence of energy transfer of electron excitation is considered. It is shown that a change in the functional dependence of the probability of Furster energy transfer leads to new characteristic dependences in the decay kinetics of impurity luminescence in layered structures.

Keywords: energy transfer, kinetics of exciton luminescence, three-layer systems.

\section{Анотація \\ ТЕОРІЯ ПЕРЕНОСУ ЕНЕРГІЇ ЕЛЕКТРОННОГО ЗБУДЖЕННЯ: КІНЕТИКА ЕКСИТОННОӤ ЛЮМІНЕСЦЕНЦІЇ В ТРЬОХШАРОВИХ СИСТЕМАХ}

Сергій І. Покутній

Розглянуто кінетику затухання екситонної люмінесценції домішкових молекул, які знаходяться в трьохшаровій системі поблизу пограничних діелектричних середовищ, при наявності переносу енергії електронного збудження між ними. Показано, що зміна функціональної залежності ферстеровської ймовірності переноса енергії приводить до нових характерних залежностей в кінетиці затухання домішкової люмінесценції шаруватих систем.

Ключові слова: перенос енергії, кінетика екситонної люмінесценції, трьохшарові системи. 


\title{
Аннотация \\ ТЕОРИЯ ПЕРЕНОСА ЭНЕРГИИ ЭЛЕКТРОННОГО ВОЗБУЖДЕНИЯ: КИНЕТИКА ЭКСИТОННОЙ ЛЮМИНЕСЦЕНЦИИ В ТРЕХСЛОЙНЫХ СИСТЕМАХ
}

\author{
Сергей И. Покутний
}

Рассмотрена кинетика затухания экситонной люминесценции примесных молекул, которые расположены в трехслойной системе вблизи граничных диэлектрических сред, при наличии переноса энергии электронного возбуждения между ними. Показано, что изменение функциональной зависимости ферстеровской вероятности переноса энергии приводит к новым характерным зависимостям в кинетике затухания примесной люминесценции слоистых систем.

Ключевые слова: перенос энергии, кинетика экситонной люминесценции, трехслойные системы.

\section{Introduction}

In inhomogeneous media (heterogeneous system, biological membranes) the problem of energy transfer of electron excitation (ETEE) on the surface is of fundamental importance [1]. ETEE in inhomogeneous systems is widely used for the development of various luminophors, scintillators, and also of materials used in quantum electronics (crystal and solutions for Lasers [2 to 4].

One of the reasons stimulating the investigation of the migration of electron excitation energy in layered media is the study of photosynthesizing systems aimed at a subsequent development of their artificial analog [5] and also the possible role of migration of excitons in biological systems [5 to10] and in chemical reactions [1].

As the ETEE theory for inhomogeneous systems is lacking it seems interesting to develop a theory of energy transfer for the case of inhomogeneous media. In [12] we advanced an ETEE theory for an inhomogeneous medium which is a layered structure. As a result the Forster formula [12] was generalized for the energy transfer probability $W(\vec{R})(\vec{R}$ is the distance between donor and acceptor molecules) and it was shown that at different intervals of distances $W(\vec{R})$ has a different power dependence on $\vec{R}$.

In the present paper on the basis of the results obtained in [12] for $W(\vec{R})$ macroscopic consequences of the energy transfer are studied, e. g. the decay laws for donor luminescence

\section{Probability of Energy Transfer in Inhomoge- neous Media}

In [12] we considered a three-layer system as a typical and simple example of an inhomogeneous medium. This system consisted of a thin film of thickness $L$ and with dielectric constant $\varepsilon_{2}$ surrounded by two semi-infinite media dielectric constants $\varepsilon_{1}$ and $\varepsilon_{3}$. The energy transfer between the two molecules was studied with their different positions to the interfaces.

The coordinate system in [12] was chosen so that the semi-space at $Z<0$ was filled by the medium with $\varepsilon_{1}$ and at $Z>L$ by that with $\varepsilon_{3}$ thus limiting the film with $\varepsilon_{2}$ at $0<Z<L$. One of the interacting molecules was on the $Z$-axis at the point with the coordinates $\left(0, Z_{1}(i)\right)$ in medium $i$, and the other in medium $j$ at the point $\left(\vec{p}, Z_{2}(j)\right)$. The interaction arising between the dipole moments of the transition of the donor $\vec{p}_{1}\left(0, Z_{1}(i)\right)$ and the acceptor $\vec{p}_{2}$ $\left(0, Z_{1}(j)\right)$ depends not only on the distance between the molecules $\vec{R}$, but also on their distance from the interface of the media $Z_{1}(i),\left(L-Z_{1}(i)\right),\left(L-Z_{2}\right.$ $(j)), Z_{2}(j)$.

The latter circumstance is due to the fact that in the case of inhomogeneous media the local field created by polarization induced by the dipoles also makes a contribution to the interaction of dipole moments of the donor $\vec{p}_{1}$ and the acceptor $\vec{p}_{2}$, in additions to direct dipole-dipole interaction.

It should be stressed that both impurity molecules and the matrix molecules are donors and acceptors of energy.

In accordance with [1] we assume that donor and acceptor molecules are oriented chaotically. Averaging the probability of the energy transfer over all orientations of dipole moments of molecules we obtain an expression $W_{(i \mid j)}(\vec{R})$ for the probability of the energy transfer described by simple analytical expressions in two limiting cases [12]: 


$$
\begin{gathered}
|C / S|=\mid\left(\left(\varepsilon_{2}-\varepsilon_{1}\right) /\left(\varepsilon_{2}+\varepsilon_{1}\right)\right) \times \\
\times\left(\left(\varepsilon_{2}-\varepsilon_{3}\right) /\left(\varepsilon_{2}+\varepsilon_{3}\right)\right) \mid<<1, \\
1-|C / S|<<1 .
\end{gathered}
$$

Conditions (1) corresponds to the situation when $\varepsilon_{2}$ of the film differs slightly from $\varepsilon_{1}, \varepsilon_{3}$ of one of the media. In case (2) $\varepsilon_{2}$ of the film differs strongly from the dielectric constants of its surrounding media. Within the framework of the Forster theory [1, 12] the probability $W_{(i \mid j)}(\vec{R})$ has the following form if (1) is fulfilled:

$$
W_{(i \mid j)}(\vec{R})=\frac{3}{2} \frac{\tilde{R}_{0}^{6}(i \mid j)}{R^{6}} \tau^{-1} .
$$

In this case the effective radius of the transition ${ }^{1}$ is written in analogy with [1] as

$$
\begin{gathered}
\tilde{R}_{0}^{6}(i \mid j)=\frac{3 \eta_{0}}{2(2 \pi)^{5} \varepsilon_{i}^{2}} \int \frac{d v}{v^{4}} \times \\
\times \int_{0}^{\pi} \int_{0}^{\pi} d \Theta_{1} d \Theta_{2} \chi^{2}(i \mid j) F\left(v, Z_{1}(i), \Theta_{1}\right) \times \\
\times \sigma\left(v, Z_{2}(j), \Theta_{2}\right) ; \Theta_{1}=\vec{p}_{1} \vec{R}, \Theta_{2}=\vec{p}_{2} \vec{R} .
\end{gathered}
$$

Here $\chi^{2}(i \mid j)$ is the orientation factor which determines the dependence of $W_{(i \mid j)}(\vec{R})$ on the orientation of dipole moments of the donor $\vec{p}_{1}$ and the acceptor $\vec{p}_{2}$ relative to the vector $\vec{R}$ separating the molecules, $\tau$ is the donor lifetime, $F(x)$ the normalized radiation spectrum of the donor, $\sigma(x)$ the effective absorption cross-section of the acceptor ${ }^{2}$ (in $\left.\mathrm{cm}^{2}\right), \eta_{0}$ the quantum output of donor luminescence, $v$ the frequency of dipole moments of donor and acceptor transitions (in $\left.\mathrm{cm}^{-1}\right)$. The value $\chi^{2}(i \mid j)$ is a function of $\chi^{2}(i \mid j)=f\left(Z_{1}(i), Z_{2}(j), \Theta_{1}, \Theta_{2}, R\right)$ and is determined for each specific case in [12]. Consider now the situation determined by (2). In this case within the interval of distances

$$
R<<2 L,
$$

${ }^{1}$ The value $\tilde{R}_{0}(i / j)$ becomes the bulk Forster radius $R_{0}$ [1] both at $\varepsilon_{1}=\varepsilon_{2}=\varepsilon_{3}$ and at $L \rightarrow \infty$.

${ }^{2}$ Below we assume that the distance from the molecule to the interface exceeds appreciably the lattice constant $a$. In this situation we may neglect a change in the radiation and absorption spectra of the molecules caused by the dependence of these spectra on the coordinates of the donor $Z_{1}(i)$ and the acceptor $Z_{2}(j)$. the transfer probability $W_{(i \mid j)}(\vec{R})$ is determined by (3) and (4). Within the interval of distance

$$
2 L \ll R \ll l_{e f f}=\frac{2 L}{|\ln (C / S)|},
$$

the function $W_{(i \mid j)}(\vec{R})$ becomes [12]:

$$
W_{(i \mid j)}(\vec{R})=\frac{3}{2}\left(\frac{r_{0}(i \mid j)}{R}\right)^{4} \tau^{-1} .
$$

Here $r_{0}(i \mid j)$, i. e. the effective radius of energy transfer within the interval of distances (6) is expressed in terms of $\tilde{R}_{0}(i \mid j)$ (4) as follows [12]:

$$
r_{0}^{4}(i \mid j)=D(i \mid j)\left(\frac{\tilde{R}_{0}(i \mid j)}{L}\right)^{2} \tilde{R}_{0}^{4}(i \mid j),
$$

where the coefficient $D(i \mid j)$ are: $D(2 \mid 2)=2^{-1}$ at $\varepsilon_{2} \gg \varepsilon_{1}, \varepsilon_{3}$ and $D \quad(2 \quad \mid \quad 2)=$ $=8^{-1}\left[1+\left(\varepsilon_{3} / \varepsilon_{2}\right)+\left(\varepsilon_{1} / \varepsilon_{2}\right)\right]^{-2} \quad$ at $\quad \varepsilon_{2}<<\varepsilon_{1}, \varepsilon_{3}$; $D(2 \mid j)=8^{-1}\left[1+\left(\varepsilon_{j} / \varepsilon_{2}\right)\right]^{-2}$ at $j=1,3$; $D(i \mid j)=8^{-1} \varepsilon_{i}^{4} \varepsilon_{2}^{-4} \varepsilon_{k}^{-4}\left(\varepsilon_{3}+\varepsilon_{1}\right)^{4}$ at $i=j=1$ index $k=3$, and at $i=j=3$ index $k=1$. If the condition imposed by the restriction of the film thickness $L$ is fulfilled [12],

$$
L<\tilde{R}_{0}(i \mid j),
$$

the effective radius of the transfer $r_{0}(i \mid j)$ (8) in some cases can exceed $(i \mid j)=(2 \mid 2),(i \mid j)=(2 \mid 1)$, $(i \mid j)=(2 \mid 3)$ at $\varepsilon_{2} \gg \varepsilon_{1}, \varepsilon_{3} ;(i \mid j)=(1 \mid 1),(i \mid j)=$ $=(3 \mid 3)$ at $\varepsilon_{2} \ll<\varepsilon_{1}, \varepsilon_{3}$ or be less (or of the order) $(i \mid j)=(2 \mid 2),(i \mid j)=(2 \mid 1)$ and $(i \mid j)=$ $=(2 \mid 3)$ at $\varepsilon_{2}<<\varepsilon_{1}, \varepsilon_{3} ;(i \mid j)=(1 \mid 1)$ and $(i \mid j)=$ $=(3 \mid 3)$ at $\varepsilon_{2} \gg \varepsilon_{1}, \varepsilon_{3}$ of the value $\tilde{R}_{0}(i \mid j)$ (4) [12], whose magnitude is close to that of the conventional Forster radius $R_{0}$ in the $i$-th medium [1] (see footnote 3).

Within the interval of distances

$$
l_{\text {eff }}<<R \text {, }
$$

the probability of the transfer $W_{(i \mid j)}$ is described by (3) and (4) in which the orientation factor $\chi^{2}(i \mid j)=$ $=f\left(\varepsilon_{1}, \varepsilon_{2}, \varepsilon_{3}, \Theta_{1}, \Theta_{2}\right)$ is determined for each particular case in [12]. 


\section{Decay Law for Donor Luminescence in a Three-Layer System}

In order to obtain macroscopic consequences of the ETEE it is necessary to average the transfer probability over the totality of molecules of energy donors and acceptors. In accordance with [1] we consider a "rigid" solution in a solvent transparent in the given spectral region. We suppose in this case that during the lifetime of the excited state mutual distances and orientation of molecules will not change. Such "rigid" solutions may be both crystalline and amorphous. We also assume that the concentration of excited molecule donors is very small [1].

The time of energy transfer is determined by

$$
t<W_{(i \mid j)}^{-1}(\vec{R}),
$$

and, depending on the intervals of distances (5), (6) and (10), there exist also the corresponding time intervals where the transfer probability has a different power dependence on $R$. In this connection the decay law for donor luminescence (DLDL) $n(t)$ in the three-layer system under consideration is described, generally speaking, by different functional dependences on $(t / \tau)$ over different time intervals. Within the framework of the above approximation, assuming that donor and acceptor molecules have statistically uniform spatial distributions with equiprobable orientations of their dipole moments and taking into account that the number of donors $n(t)$ decreases not only due to the transfer but also by spontaneous emission, we obtain the DLDL [1]

$$
n(t)=\frac{n_{0}}{L} \int_{l_{1}}^{l_{2}} d Z_{1}(i) \exp \left[-\frac{t}{\tau}-H\left(t, Z_{1}(i)\right)\right]
$$

where $n_{0}$ is the concentration of excited molecule donors at $t=0$.

When calculating the function $H\left(t, Z_{1}(i)\right)$ one must take into account that the rate of the transfer depends not only on the distance $R$ but also on the mutual orientation of dipole moments.

In this connection when calculating $H\left(t, Z_{1}(i)\right)$ we assume that the dipole moment of the donor is directed along the $Z$-axis and we shall average over the orientations of the acceptors.

We write the function

$$
H=c \iint_{V}\left[1-\exp \left(-W_{(i \mid j)}(\vec{R}) t\right)\right] d V d \Omega,
$$

in which $c$ is the concentration of the acceptors, $V$ the limited volume containing the acceptor molecules, $d \Omega$ the element of the body angle that determines the direction of the acceptor dipole moment. Integration in (13) is carried out over the whole volume and all directions of dipole moment and radius vector. In (12) integration is made over the region of molecule donors which in the three-layer system under consideration is, generally speaking, limited.

\section{Decay Law of Luminescence for Donor Molecules near the Interface in a Three-Layer System}

In $[12,13]$ DLDL $_{\mathrm{s}}$ were obtained for the case when donor and acceptor molecules are in the bulk of the film. In the present work, on the bases of the results obtained in [12] for $W(\vec{R}), \mathrm{DLDL}_{\mathrm{s}}$ are studied for donor molecules near the interface in a threelayer system.

Consider the situation when donor molecules are either in the film or in the media surrounding the film and acceptor molecules are outside the film. To calculate the function $H\left(t, Z_{1}(i)\right)$ (13), when (2) is fulfilled, the film of the thickness $L$ was divided into three regions by the radius vector of length $2 L, l_{e f f}$, and $\infty$. In this case the radius vector originated from the point of the dipole moment of the donor $\vec{p}_{1}$, directed along the $Z$-axis. The expression for $H\left(t, Z_{1}(i)\right)$ is not given here because it is too cumbersome.

Substituting the expressions for the probability of energy transfer $W_{(i \mid j)}(3)$ into (13) with subsequent calculation of the function $H\left(t, Z_{1}(i)\right)$ and its substitution (12) we obtained DLDL over the time interval

$$
0<\frac{t}{\tau}<\frac{2}{3}\left(\frac{L}{2 \tilde{R}_{0}(i \mid j)}\right)^{6},
$$

that corresponds to the interval of distances (5) in the following form [16]:

$$
n(t)=n_{0} \exp \left[-\frac{t}{\tau}-q_{0}(i \mid j)\left(\frac{t}{\tau}\right)^{1 / 2}-q_{1}(i \mid j) \frac{t}{\tau}\right],
$$

where the coefficients $q_{0}(i \mid j)=0, q_{1}(i \mid j)=$ $=0.12 \pi^{3 / 2} \tilde{C}(i \mid j) \quad \tilde{L}^{3}(i \mid j)$ at $i=2, \quad j=1,3$; if $i=j=1$, or $i=j=3$, then $q_{0}(i \mid j)=$ $=1.12 \pi^{3 / 2} \tilde{C}(i \mid j), q_{1}(i \mid j)=0.83 \pi^{3 / 2} \quad \tilde{C} \tilde{L}^{3}(i \mid j)$. In the case when $i=1$ and $j=3$ the coefficients 
$q_{0}(1 \mid 3)=0$ and $q_{1}(1 \mid 3)=8 \times 10^{-2} \pi^{3 / 2} \quad \tilde{C}(1 \mid 3) \times$ $\times \tilde{L}^{3}(1 \mid 3)$. The dimensionless parameters $\tilde{C}(i \mid j)$ and $\tilde{L}(i \mid j)$ equal, respectively: $\tilde{C}(i \mid j)=C \tilde{R}_{0}^{3}(i \mid j)$, $\tilde{L}(i \mid j)=\tilde{R}_{0}(i \mid j) / L$. At times

$$
\frac{2}{3}\left(\frac{L}{2 \tilde{R}_{0}(i \mid j)}\right)^{6}<\frac{t}{\tau}<\frac{2}{3}\left(\frac{2 L}{\tilde{R}_{0}(i \mid j)}\right)^{6},
$$

the decay law $n(t)$ is determined by the expression [16]:

$$
\begin{aligned}
& n(t)=n_{0} \exp \left[-\frac{t}{\tau}-q_{0}(i \mid j)\left(\frac{t}{\tau}\right)^{1 / 2}-q_{1}(i \mid j) \frac{t}{\tau}\right]+ \\
& +n_{0} \exp \left[-\frac{t}{\tau}-q_{2}(i \mid j)\left(\frac{t}{\tau}\right)^{1 / 2}+q_{3}(i \mid j) \frac{t}{\tau}\right]
\end{aligned}
$$

where the coefficients are

$q_{2}(i \mid j)=0,2(1+5 \sqrt{3 D(i \mid j) / 8}) \pi^{3 / 2} \tilde{C}(i \mid j) ; q_{3}(i \mid j)=$ $2 \times 10^{-2}\left[1+9 \ln ^{2}(C / S) / 4\right] \pi^{3 / 2} \tilde{C}(i \mid j) \tilde{L}^{3}(i \mid j)$ at $i=2, j=1,3$. In the case when $i=j=1$, or $i=j=3$ the coefficients

$q_{2}(i \mid j)=0,1 \pi^{3 / 2} \tilde{C}(i \mid j), q_{3}(i \mid j)=0.1 \pi^{3 / 2} \tilde{C}(i \mid j) \tilde{L}^{3}(i \mid j)$, but if $i=1$ and $j=3$, then $q_{2}(1 \mid 3)=0.2 \pi^{3 / 2} \tilde{C}(1 \mid 3)$, $q_{3}(1 \mid 3)=-10^{-2} \pi^{3 / 2} \tilde{C}(1 \mid 3) \tilde{L}^{3}(1 \mid 3)$.

Over the time interval

$$
\begin{gathered}
\frac{2}{3}\left(\frac{2 L}{\tilde{R}_{0}(i \mid j)}\right)<\frac{t}{\tau}< \\
<\left[6 D(i \mid j) \ln ^{4}(C / S)\right]^{-1}\left(\frac{2 L}{\tilde{R}_{0}(i \mid j)}\right)^{6}
\end{gathered}
$$

that corresponds to the interval of distances (6), DLDL can be represented as follows:

$$
\begin{aligned}
& n(t)=n_{0} \exp \left[-\frac{t}{\tau}-q_{2}(i \mid j)\left(\frac{t}{\tau}\right)^{1 / 2}+q_{3}(i \mid j) \frac{t}{\tau}\right] \\
& -n_{0} \exp \left[-\frac{t}{\tau}-q_{4}(i \mid j)\left(\frac{t}{\tau}\right)^{1 / 2}+q_{5}(i \mid j) \frac{t}{\tau}\right]
\end{aligned}
$$

In (19) the coefficients are

$$
q_{4}(i \mid j)=\sqrt{3 D(i \mid j) / 8} \pi^{3 / 2} \tilde{C}(i \mid j) ; \quad q_{5}(i \mid j)=5 \times
$$

$\times 10^{-2} D(i \mid j) \ln ^{2}(C / S) \pi^{3 / 2} \tilde{C}(i \mid j) \tilde{L}^{3}(i \mid j)$ at $i=2$ and $j=1,3$; if $i=j=1$ or $i=j==3$, then the coefficients $q_{4}(i \mid j)=0, q_{5}(i \mid j)=$ $=-4 \times 10^{-2} D(i \mid j) \pi^{3 / 2} \tilde{C}(i \mid j) \tilde{L}^{3}(i \mid j) ;$ at $i=1, j=3$; $q_{4}(1 \mid 3)=0$ and $q_{5}(1 \mid 3)=-0.2 D(1 \mid 3) \pi^{3 / 2} \tilde{C}(1 \mid 3) \times$ $\times \tilde{L}^{3}(1 \mid 3)$. The parameter $D(i \mid j)$ present in the coefficients in (17) and (19) was determined before in (8).

Over the time interval corresponding to the interval of distances (10) the value $t / \tau>1$, therefore the decay law for $n(t)$ over this interval is not considered, as the latter can easily be shown to make only a negligibly small contribution to the decay law compared to the appropriate contributions $n(t)$ (17) and (19).

When (1) is fulfilled over the time interval $0<t / \tau<1$ the decay law for $n(t)$ is determined by the following formula [16]:

$n(t)=n_{0} \exp \left[-\frac{t}{\tau}-q_{0}(i \mid j)\left(\frac{t}{\tau}\right)^{1 / 2}-q_{1}(i \mid j) \frac{t}{\tau}\right]$

where the coefficients $q_{0}(i \mid j) \approx 1.12 \pi^{3 / 2} \tilde{C}(i \mid j)$, $q_{1}(i \mid j) \approx 0.83 \pi^{3 / 2} \tilde{C}(i \mid j) \tilde{L}^{3}(i \mid j)$.

Over the time interval

$$
0<\left(\frac{t}{\tau}\right)^{1 / 2}<<\frac{q_{0}(i \mid j)}{q_{1}(i \mid j)-1}
$$

(20) includes the decay law in a homogeneous medium [1]

$$
n(t)=n_{0} \exp \left[-\frac{t}{\tau}-q_{0}\left(\frac{t}{\tau}\right)^{1 / 2}\right]
$$

where $q_{0} \approx 1.12 \pi^{3 / 2} \tilde{C}$.

Let us analyze the decay laws for $n(t)(15),(17)$, and (19) for the case when (2) is fulfilled. The case when the indices $(i \mid j)=(2 \mid 1)$ or $(i \mid j)=(2 \mid 3)$ corresponds to the situation when donor molecules are in the film and acceptor molecules are outside the film in the media with $\varepsilon_{1}$ or $\varepsilon_{3}$. In the second case $(i \mid j)=(1 \mid 3)$ donor molecules are in the medium with $\varepsilon_{1}$ and the acceptor molecules are in the medium with $\varepsilon_{3}$. In both cases in contrast to the situation when molecules are only on the bulk of the film $[14,15]$ there is no time interval over which $n$ $(t)$ coincides with the appropriate decay law in the homogeneous medium (22). The latter is caused by the fact even at small times (14) the energy transfer occurs over a distance of the order of one of the characteristic sizes of inhomogeneities $\left(L-Z_{1}(i)\right)$ or $Z_{1}$ (i), therefore the coefficient $q_{0}(2 \mid 1)=q_{1}(2 \mid 3)=0$.

Consider the case when $(i \mid j)=(1 \mid 3)$, i. e. donor molecules are in the medium with $\varepsilon_{1}$ and the accep- 
tor molecules are in the medium with $\varepsilon_{3}$. Here, as in the previous two cases, there is no time interval where $n(t)$ would coincide with the appropriate DLDL in an inhomogeneous medium (22).

In [16] the decay laws are studied for the cases when the spatial distribution of acceptors will, generally speaking, be restricted by a film from only one side. In this connection the DLDL (19) in contrast to an appropriate law, which describes donor decays for the case when donor and acceptor molecules are in the bulk of the film $[14,15]$, will have in the exponent no terms of the form $\left(\ln q_{6}(t / \tau)^{1 / 6}, q_{2}(t / \tau)^{3 / 4}\right.$, and $q_{5}(t / \tau)^{1 / 6}$ ), caused by restriction of the spatial distribution of donors and acceptors by the film.

It should be noted that (9), due to the smallness of the value $\boldsymbol{R}_{0} \approx 5$ to $10 \mathrm{~nm}[1,13]$, is fulfilled only for three-layer systems consisting of thin films $L<\tilde{R}_{0}$. Therefore, the terms in (15), (17), (19), and (20) with the coefficients $q_{1}(i \mid j), q_{3}(i \mid j)$, and $q_{5}(i \mid j)$, due to the geometry of the inhomogeneous system, will make the main contribution to the appropriate $\mathrm{DLDL}_{\mathrm{s}}$ for $\boldsymbol{n}(\boldsymbol{t})$ when (9) is fulfilled [16].

\section{Conclusions}

In an inhomogeneous medium radiation and absorption of a molecule depend on its coordinate: therefore, experimental values of the coefficients of absorption and luminescence of molecules in an inhomogeneous medium are integrals of local characteristics of radiation or absorption. Consequently, the characteristics distance $\tilde{R}_{0}$ (4) cannot be expressed in terms of experimental values of the spectra. In this connection a comparison of our DLDL for $n(t)$ with the curves for $n(t)$ taken from experiments on resonance quenching of donors allows one to determine the value $\tilde{R}_{0}$ in layered structures.

As a three-layer structure a system consisting of a biological membrane surrounded by water can be chosen. (This system is described by (1).). The decay laws for $n(t)$ found in such systems can be used to improve the technique of luminescent probes [6 to 8].

As three-layer systems in which (2) is fulfilled, apparently one can use thin semiconductor or dielec- tric films of thickness $\boldsymbol{L}<5 \mathbf{n m}$ activated by rareearth ions $[2,3]$ deposited on dielectric substrates (in this case $\varepsilon_{2}$ of the film differs strongly from $\varepsilon_{1}, \varepsilon_{3}$ of the substrates). Investigation of energy transfer processes in such three-layer structures is a rather urgent problem associated with the search for laser active materials $[2,4,11]$.

\section{Referents}

1. Agranovich V. M., Galanin M. D. Perenos energii elektronogo vozbuzhdeniya $\mathrm{v}$ kondensirovannikh sredakh. - Nauka, Moscow: 1978. — 384 p.

2. Alimov O. K., Basiev T. T. // Sov. Phys. - Zh. Eksper. Theor. Fiz. - 1977. — V. 72, №3. - P. 13121319.

3. Bandurkin G. A. Osobennosti kristallokhimii soedinenii redkozemelnikh elementov. - Nauka, Moscow: 1984. $-232 \mathrm{p}$.

4. Bagdasarov Kh. C., Danilov V. P. // Sov. Phys. Kvant. Electron. - 1978. - V. 5, №1. - P. 150155.

5. Braginskaya O. V., Efremov N. A. // Sov. Phys. Dokl. Akad. Nauk SSSR. — 1982. - V. 268, №11. - P. 1109-1111.

6. Mauzerall D. // Biophys. - 1976. - V. 16, №1. P. 87-96.

7. Wolber P., Hudson B. // Biophys. - 1979. - V. 28, №2. - P. 197-204.

8. Rybin L. B., Bradinskaya O. V. // Molec. Biol. 1980. - V. 14, №4. - P. 675-682.

9. Pokutnyi S. I. // Ukr. Fiz. Zh. - 1995. - V. 40, №10. - P. $1134-1137$.

10. Pokutnyi S. I. // Biophysics. — 1999. - V. 44, №3. - P. 388-391.

11.Zverev G. M., Garmash V. M. // Sov. Phys. - Zh. Prekl. Spektros. - 1974. - V. 21, №6. - P. 820824.

12. Efremov N. A., Pokutnyi S. I. // Phys. Sol. Stat. 1993. - V. 35, №5. - P. $575-586$.

13. Ermolaev V. L, Bodunov E. N. Bezizluchatelnii perenos energii electronogo vozbuzhdenija. Nauka, Moscow: 1977. - $311 \mathrm{p}$.

14. Pokutnyi S. I. // Sov. Phys. - Chem. Fizika. 1988. - V. 7, №4. - P. 613-615.

15. Pokutnyi S. I. // Phys. Stat. Sol. (b). - 1988. V. 147, №1. - P. $21-24$.

16. Pokutnyi S. I. // Phys. Stat. Sol. (b). - 1990. V. 157, №1. - P. 221-227. 